\title{
Dramatic role of critical current anisotropy on flux avalanches in $\mathrm{MgB}_{2}$ films
}

\author{
J. Albrecht, A. T. Matveev* \\ Max-Planck-Institut für Metallforschung, Heisenbergstr. 3, D-70569 Stuttgart, Germany \\ J. Strempfer, H-U. Habermeier \\ Max-Planck-Institut für Festkörperforschung, Heisenbergstr. 1, D-70569 Stuttgart, Germany \\ D. V. Shantsev, Y. M. Galperin and T. H. Johansen \\ Department of Physics, University of Oslo, P. O. Box 1048, Blindern, 0316 Oslo, Norway
}

\begin{abstract}
Anisotropic penetration of magnetic flux in $\mathrm{MgB}_{2}$ films grown on vicinal sapphire substrates is investigated using magneto-optical imaging. Regular penetration above $10 \mathrm{~K}$ proceeds more easily along the substrate surface steps, anisotropy of the critical current being $6 \%$. At lower temperatures the penetration occurs via abrupt dendritic avalanches that preferentially propagate perpendicular to the surface steps. This inverse anisotropy in the penetration pattern becomes dramatic very close to $10 \mathrm{~K}$ where all flux avalanches propagate in the strongest-pinning direction. The observed behavior is fully explained using a thermomagnetic model of the dendritic instability.
\end{abstract}

Above the lower critical field $H_{c 1}$ magnetic flux penetrates type-II superconductors in the form of quantized vortices. Usually, this penetration takes place as a regular flow leading to a smooth flux front [1]. However, in some superconducting films, e.g. of $\mathrm{MgB}_{2}$ a dramatic flux instability can occur resulting in an abrupt formation of magnetic dendrites [2]. The flux instability is believed to arise because the motion of flux lines dissipates energy leading to local heating, which results in a reduction of pinning and an enhancement of flux line mobility. This constitutes a positive feedback loop which acts as driving force for the avalanches $[3,4]$. Understanding and controlling the avalanches is important not only from the fundamental point of view, but also for applications since they represent huge magnetic noise and reduce the effective critical current density.

Recently, numerous experimental investigations have demonstrated that the dendritic instability is sensitive to external conditions. The instability was found to disappear above threshold values of temperature [2], applied magnetic field [5], and also for sufficiently small sample dimensions [6]. Also covering $\mathrm{MgB}_{2}$ films with a layer of gold or aluminium was seen to suppress the dendrite formation [7] or change their propagation direction [8].

To what extent the dendritic instability is sensitive to the internal structure and flux pinning properties of the superconducting film is an important and yet open question. A recent thermomagnetic model predicts that pinning strength represented by the critical current density $j_{c}$ controls the onset of the dendritic instability $[9,10]$. In this paper we show that $\mathrm{MgB}_{2}$ films with slightly anisotropic $j_{c}$ can exhibit large anisotropy in the avalanche activity, in full agreement with the model. The result gives direct evidence that the microstructure of chemically homogeneous films grown on vicinal substrates defines a direction for dendritic flux propagation, which surprisingly turns out to be perpendicular to the lowest pinning direction.
Substrate-induced modifications of the pinning properties have successfully been obtained previously in hightemperature superconducting films grown on vicinal substrate surfaces $[11,12]$, and on artificially patterned substrates $[13,14]$. To create anisotropic pinning in films of $\mathrm{MgB}_{2}$ we used $\mathrm{r}$-cut $\mathrm{Al}_{2} \mathrm{O}_{3}$ substrates slightly miscut

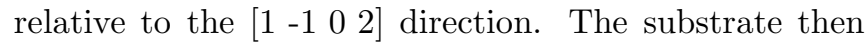
possesses a surface step structure which can be characterized by two angles $\Delta \theta$ and $\Delta \phi$, according to the sketch in Fig. 1. Shown in the graph is the result of x-ray diffraction measurements obtained from surface reflection of a substrate mounted parallel to the $\phi$-axis of a 4 -circle diffractometer. The deviation $\theta$ of the direction of the

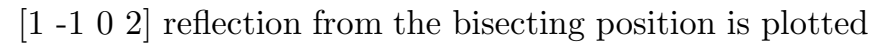
as function of the angle $\phi$. From the curve it follows that the substrate misorientation angles are $\Delta \theta / 2=0.81^{\circ}$ and $\Delta \phi=12^{\circ}$. With a step height of one unit cell $a$ of $\mathrm{Al}_{2} \mathrm{O}_{3}$, the step distance becomes $d=a / \tan (\Delta \theta / 2) \approx 27 \mathrm{~nm}$.
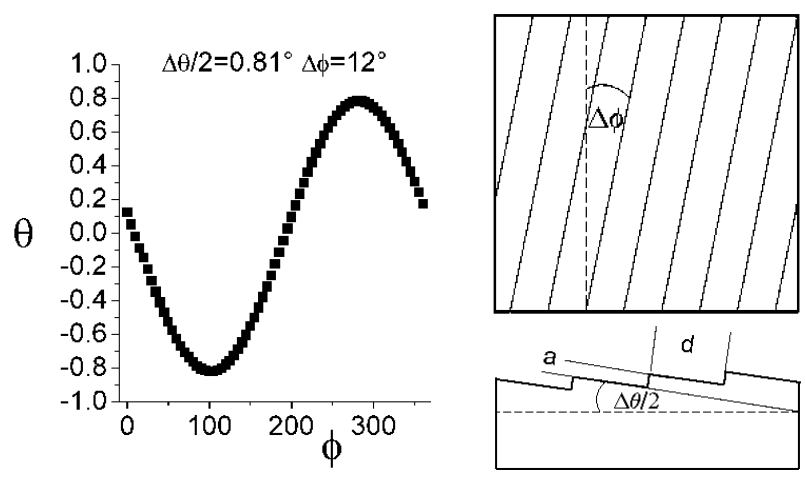

FIG. 1. (Left) X-ray diffraction data for a sapphire sub-

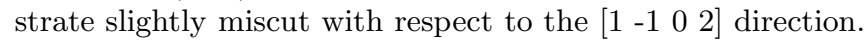
(Right) Sketch of the square substrate with surface steps defining the angles $\Delta \theta$ and $\Delta \phi$ which are determined from the plotted data. 


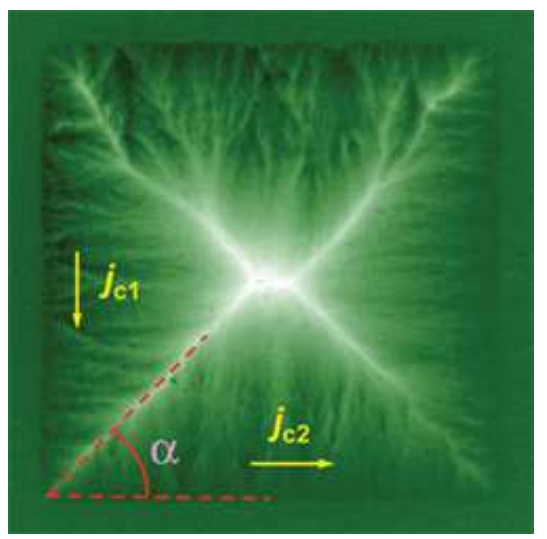

FIG. 2. Magneto-optical image of the remanent state in an anisotropic $5 \times 5 \mathrm{~mm}^{2} \mathrm{MgB}_{2}$ film at $12 \mathrm{~K}$. The angle $\alpha$ indicates the orientation of the discontinuity line which allows determination of the critical current density ratio $j_{c 1} / j_{c 2}$.

The $\mathrm{MgB}_{2}$ films were grown by sequential deposition of magnesium and boron layers using a conventional electron beam evaporation and a subsequent annealing process $[15,16]$. The films selected for this study had a lateral size of $5 \times 5 \mathrm{~mm}^{2}$ and a thickness of $200 \mathrm{~nm}$. The magnetic flux penetration into these films was investigated using the magneto-optical (MO) Faraday effect allowing direct visualization of the flux distribution [1]. In our images the brightness represents the magnitude of the flux density.

To measure the anisotropy of the critical current density the sample was first cooled to $12 \mathrm{~K}$, and then exposed to a large magnetic field which was subsequently removed. In this remanent state, seen in Fig. 2, the flux distribution develops a set of bright discontinuity lines (dlines), where the current sharply bends to adapt to the shape of the sample [17]. In an isotropic film the angle between the d-lines and the edge would be $45^{\circ}$ and the dlines would meet in the center of the square. In our samples this is not the case, as seen directly by the presence of a horizontal bright line segment in the central part of the square. Evidently, the current flowing parallel to the vertical edges gives stronger shielding than the current flowing horizontally. Assuming Bean-model behavior, the two critical current densities $j_{c 1}$ and $j_{c 2}$ are related to the d-line angle $\alpha$, see the figure, by $j_{c 1} / j_{c 2}=\tan \alpha$. Measurement of the angle gives $\tan \alpha=1.06$, i.e., a $6 \%$ anisotropy. Comparing with the direction of the steps on the substrate surface, see Fig. 1, the larger value of $j_{c 1}$ represents a pinning force enhancement for flux moving perpendicular to the surface steps.

Shown in Fig. 3 is the flux distribution after applying a $8 \mathrm{mT}$ magnetic field to the $\mathrm{MgB}_{2}$ film initially zerofield-cooled to the slightly lower temperatures. Here one should expect the flux to penetrate in the form of dendritic avalanches [2], which indeed is what we observe. At $8 \mathrm{~K}$ the avalanches are seen to enter the film evenly from all the edges. At $10 \mathrm{~K}$, however, the penetration pattern suddenly becomes strongly anisotropic; flux dendrites develop from the vertical edges only, whereas from the top and bottom edges the penetration is smooth. In other words, the flux dendrites propagate predominantly in the direction perpendicular to the substrate surface steps, i.e., the direction of the stronger flux pinning. It is indeed highly counterintuitive that the flux prefers to move perpendicular rather than parallel to the linear defects introduced in the sample.

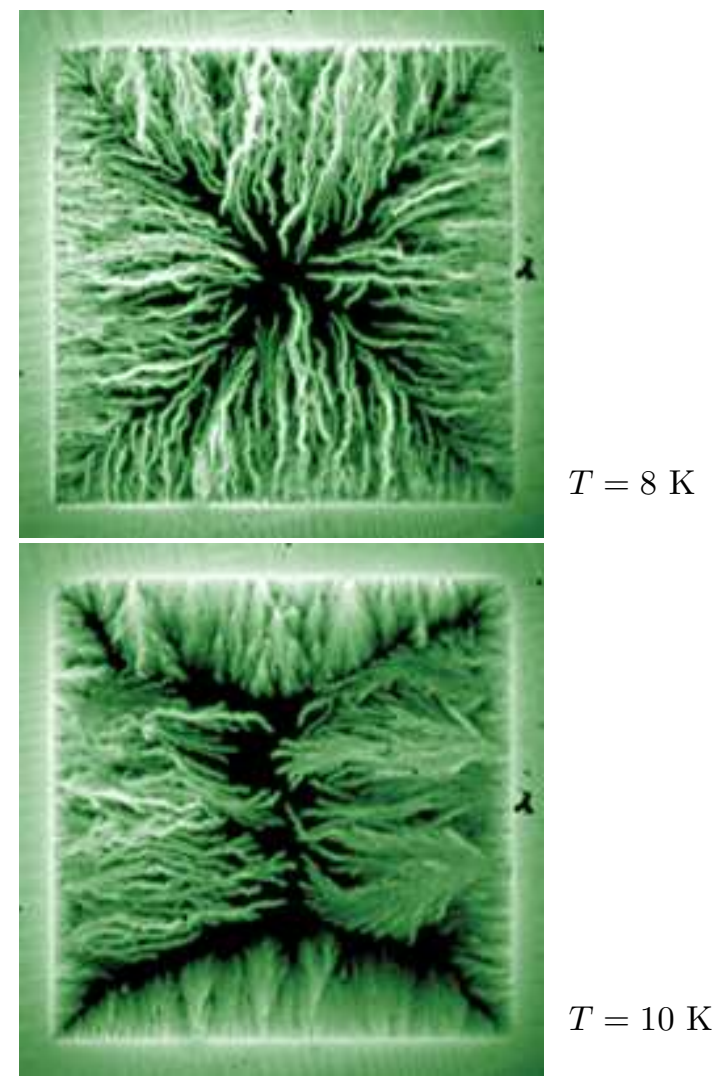

FIG. 3. Flux penetration into the same $\mathrm{MgB}_{2}$ film as in Fig. 2, but at lower temperatures and an applied field of $16 \mathrm{mT}$. At $8 \mathrm{~K}$ the flux pattern is dendritic and isotropic, while at $10 \mathrm{~K}$ dendritic avalanches are formed only along one direction.

A quite similar behavior was observed for the penetration behavior during field descent, see Fig. 4. The images show the remanent state after applying a maximum field of $\mu_{0} H=0.2 \mathrm{~T}$. Again at $8 \mathrm{~K}$, the penetration is isotropic only now the dendrites appear dark since they consist of antiflux surrounded by annihilation zones. At $10 \mathrm{~K}$ the dendrites propagate also now along the horizontal direction dictated by the microstructural anisotropy. 


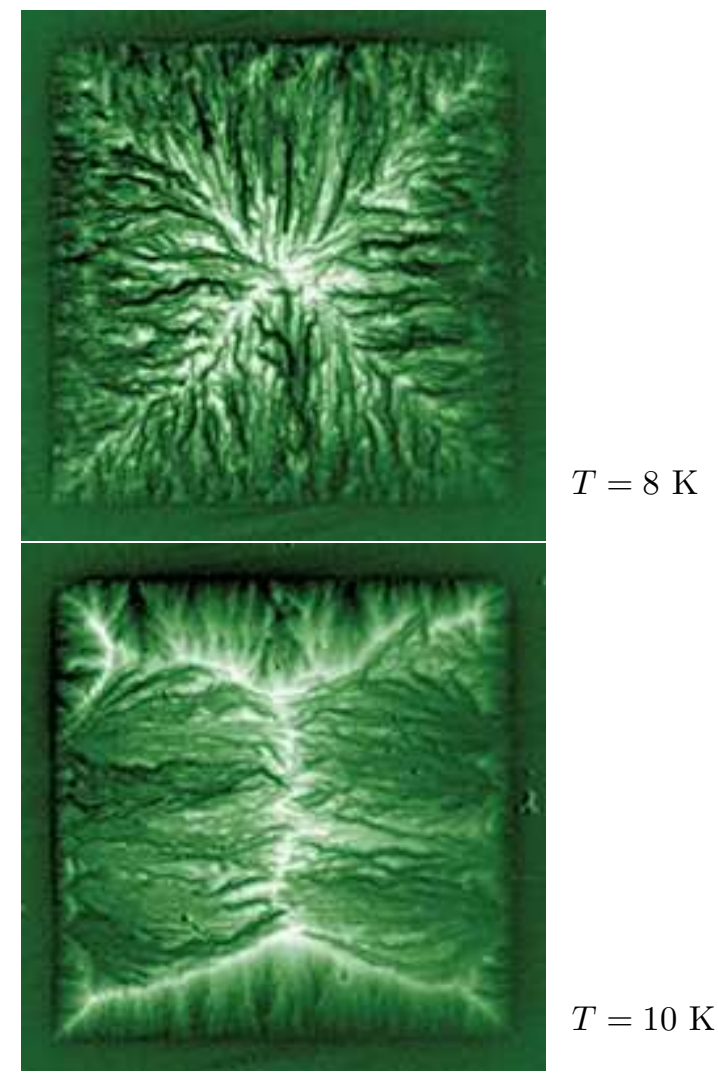

FIG. 4. Remanent states of the anisotropic $\mathrm{MgB}_{2}$ film. The flux pattern is isotropic at $8 \mathrm{~K}$, but strongly anisotropic at $10 \mathrm{~K}$, similar to the virgin penetration behavior in Fig. 3.

All these experiments show the remarkable fact that an anisotropy in $j_{c}$ as small as $6 \%$ can lead to a dramatic anisotropy in the avalanche activity near $10 \mathrm{~K}$, and only very near this temperature. This behavior can be understood using results of a recent model of the dendritic instability developed in Refs. $[9,10]$. The model is based on stability analysis of the thermal diffusion and Maxwell equations in a long and thin superconducting strip thermally coupled to a substrate. Initially, the strip is placed in an increasing perpendicular magnetic field, and a Bean-like critical state is formed in the flux penetrated region. This state can become unstable with respect to perturbations in the magnetic field and temperature, and under some conditions a fastest growing perturbation has a non-zero wave vector along the film edge. This means that an instability will develop in the form of narrow fingers perpendicular to the edge - a scenario closely resembling the observed dendritic flux behavior. The threshold flux penetration depth, $\ell^{*}$, when the superconducting strip first becomes unstable, is given by Eq. (25) of Ref. [9], which can be written as

$$
\ell^{*}=\frac{\pi}{2} \sqrt{\frac{\kappa T^{*}}{j_{c} E}}\left(1-\sqrt{\frac{2 h_{0} T^{*}}{n d j_{c} E}}\right)^{-1} .
$$

Here, $d$ is the film thickness, $T^{*} \equiv-\left(\partial \ln j_{c} / \partial T\right)^{-1}$, $\kappa$ is the thermal conductivity, and $h_{0}$ is the coefficient of heat transfer from the superconducting film to the substrate. The parameter $n$ characterizes the nonlinearity of the current-voltage curve of the superconductor, $n=\partial \ln E / \partial \ln j \gg 1$.

The threshold field, $H_{t h}$, when the first dendrite invades the film is obtained by combining (1) with the Bean model expression,

$$
H=\frac{j_{c} d}{\pi} \operatorname{arccosh}\left(\frac{w}{w-\ell}\right)
$$

which connects the the applied field with the flux penetration depth $\ell$ for a long thin strip of width $2 w$ [18].

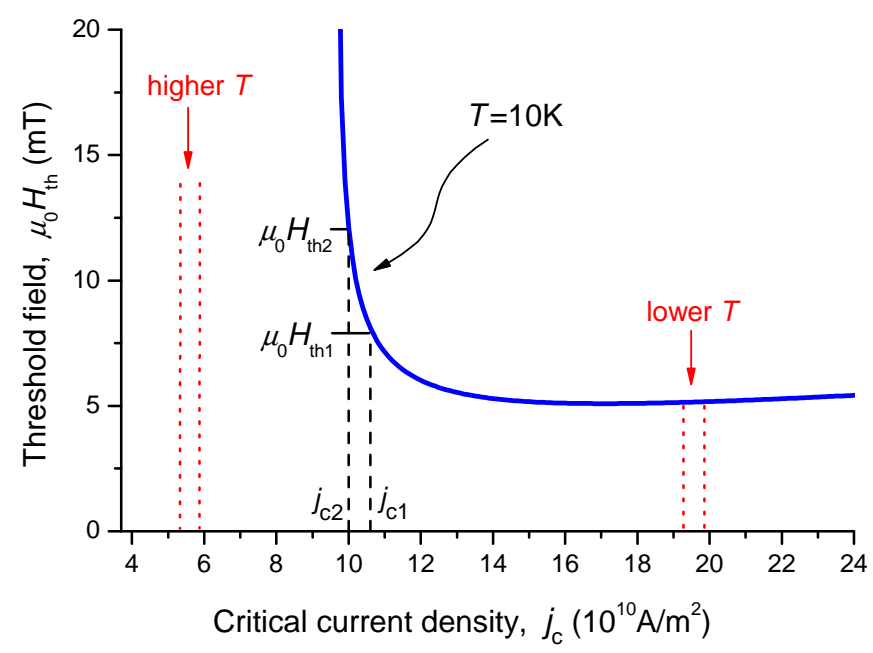

FIG. 5. Threshold field for the dendritic instability as a function of the critical current density, calculated using (1) and (2). Two dashed lines show the values of $j_{c}$ in an $\mathrm{MgB}_{2}$ film at $10 \mathrm{~K}$ for the directions along and across the substrate steps. Large difference in the corresponding $H_{t h}$ 's implies a strong anisotropy of the dendritic avalanches specifically at $T=10 \mathrm{~K}$.

Fig. 5 shows the dependence of $H_{t h}$ on $j_{c}$ calculated using Eqs. (1) and (2) assuming $\kappa T^{*} / E=140 \mathrm{~A}$ and $h_{0} T^{*} / n E=9230 \mathrm{~A} / \mathrm{m}$ (which can mean e.g. $T^{*}=10 \mathrm{~K}$, $E=10 \mathrm{mV} / \mathrm{m}, \kappa=0.14 \mathrm{~W} / \mathrm{Km}, n=30$, and $h_{0}=$ $\left.280 \mathrm{~W} / \mathrm{Km}^{2}\right)$. The graph shows that for high critical currents the threshold field is weakly dependent on $j_{c}$. However, when $j_{c}$ becomes smaller, the expression in the brackets of Eq. (1) approaches zero, and $H_{t h}$ diverges. When $j_{c}$ drops below a certain value, the dendritic instability is absent no matter how large field is applied.

The critical current density at $10 \mathrm{~K}$ can be estimated using Eq. (2) from the flux penetration depth just before the avalanche behavior sets in. We then find $j_{c 2}=1 \times 10^{11} \mathrm{~A} / \mathrm{m}^{2}$, and $j_{c 1}$ larger by $6 \%$. These critical current values found from MO images agree with results obtained by SQUID measurements [16]. The two values for $j_{c}$ are indicated by two dashed lines in Fig. 5 . 
Despite the small difference between $j_{c 1}$ and $j_{c 2}$, the corresponding threshold fields $H_{t h 1}$ and $H_{t h 2}$ differ significantly. When the increasing applied field reaches the lowest of the two threshold fields, $H_{t h 1}$, the dendrites should appear from the sides where $j_{c}$ is the highest. This is exactly what one can see from Fig. 3 (bottom). Interestingly, this penetration pattern dominated by dendrites shows an inverted anisotropy compared to the critical state pattern in Fig. 2.

As the applied field is further increased and reaches $H_{t h 2}$, one could expect that dendrites appear also from the top and bottom edges. However, this does not happen experimentally, as can be seen from the $10 \mathrm{~K}$ images. The reason probably is the dramatic disturbance in the current flow created by the dendritic structures formed at the smaller fields. They fill almost the whole interior of the film, thus lowering the edge fields substantially, which prevents invasion of new dendrites.

At a higher temperature, the critical current density becomes smaller, i.e., we "move" to the left along the $x$ axis in Fig. 5, and enter the stable region. This is again in full agreement with experiment, where we do not observe dendritic avalanches above $10 \mathrm{~K}$. Conversely, by lowering the temperature we move to the right, where $H_{t h}$ is almost independent of $j_{c}$. Hence, the dendritic avalanches should penetrate evenly from all four sides of the square film. This is exactly what we find at $8 \mathrm{~K}$, see Fig. 4 (top), and at lower $T$. [19]

Note, that the $H_{t h}\left(j_{c}\right)$ curve is slowly rising at large $j_{c}$. It means that at very low $T$ one should expect the opposite kind of anisotropy in the dendritic growth. Namely, the dendrites should first appear from the sides where the regular flux penetration is deeper. However, in order to observe this effect one needs a sample where the anisotropy in $j_{c}$ is much stronger than the $6 \%$ in the present case.

The analysis above shows that the dendritic penetration has a pronounced anisotropy only in the narrow interval of temperatures corresponding to the steep region at the $H_{t h}\left(j_{c}\right)$ curve. For other superconducting samples this region will not necessarily be located at $10 \mathrm{~K}$, but it will always be near the threshold temperature above which the instability disappears.

In conclusion, we have investigated the magnetic flux penetration into $\mathrm{MgB}_{2}$ films grown on vicinal sapphire substrates. The resulting microstructure giving a slight anisotropy in $j_{c}$ leads to a large anisotropy in the flux patterns, but only in a narrow temperature interval near $10 \mathrm{~K}$. Here, the dendritic avalanches propagate only in the direction perpendicular to the substrate surface steps, i.e., the flux moves along the direction of largest pinning. The surprising behavior is fully explained by a thermomagnetic model for the dendritic instability.
* The current affiliation: Chemistry Department, Lomonosov MSU, 119992 Moscow, Russia

[1] Ch. Jooss, J. Albrecht, H. Kuhn, S. Leonhardt, and H. Kronmüller, Rep. Prog. Phys. 65, 651 (2002).

[2] T. H. Johansen, M. Baziljevich, D. V. Shantsev, P. E. Goa, Y. M. Galperin, W. N. Kang, H. J. Kim, E. M. Choi, M.-S. Kim, and S. I. Lee, Europhys. Lett. 59, 599 (2002).

[3] R. G. Mints and A. L. Rakhmanov, Rev. Mod. Phys. 53, 551 (1981).

[4] E. Altshuler and T. H. Johansen, Rev. Mod. Phys. 76, 471 (2004).

[5] I. A. Rudnev, D. V. Shantsev, T. H. Johansen, and A. E. Primenko, Appl. Phys. Lett. 87, 042502 (2005).

[6] D. V. Denisov et al, unpublished.

[7] M. Baziljevich, A. V. Bobyl, D. V. Shantsev, E. Altshuler, T. H. Johansen, and S. I. Lee, Physica C 369, 93 (2002).

[8] J. Albrecht, A. T. Matveev, M. Djupmyr, G. Schütz, B. Stuhlhofer, and H.-U. Habermeier, Appl. Phys. Lett. 87, 182501 (2005).

[9] D. V. Denisov, A. L. Rakhmanov, D. V. Shantsev, Y. M. Galperin, T. H. Johansen, Phys. Rev. B 73, 014512 (2006).

[10] I. S. Aranson, A. Gurevich, M. S. Welling, R. J. Wijngaarden, V. K. Vlasko-Vlasov, V. M. Vinokur, U. Welp, Phys. Rev. Lett. 94, 037002 (2005).

[11] Ch. Jooss, R. Warthmann, and H. Kronmüller, Phys. Rev. B 61, 12433 (2000).

[12] M. Djupmyr, G. Cristiani, H.-U. Habermeier, and J. Albrecht, Phys. Rev. B 72, 220507(R) (2005).

[13] J. Albrecht, S. Leonhardt, H.-U. Habermeier, S. Brück, R. Spolenak, and H. Kronmüller, Physica C 404, 18 (2004).

[14] S. Brück and J. Albrecht, Phys. Rev. B 71, 174508 (2005).

[15] S. R. Shinde, S. B. Ogale, R. L. Greene, T. Venkatesan, P. C. Canfield, S. L. Bud'ko, G. Lapertot, and C. Petrovic, Appl. Phys. Lett. 79, 227 (2001).

[16] A. T. Matveev, J. Albrecht, M. Konuma, B. Stuhlhofer, U. Starke, and H.-U. Habermeier, Supercond. Sci. Technol. 18, 1313 (2005).

[17] Th. Schuster, M. V. Indenbom, M. R. Koblischka, H. Kuhn, H. Kronmüller, Phys. Rev. B 49, 3443 (1994).

[18] E. H. Brandt, and M. Indenbom, Phys. Rev. B 48, 12893 (1993). E. Zeldov, J. R. Clem, M. McElfresh, and M. Darwin, Phys. Rev. B 49, 9802 (1994).

[19] In principle, one should also take into account that $\kappa$ and $h_{0}$ are expected to increase with temperature. It can be easily verified that for $T>10 \mathrm{~K}$ this shifts the $H_{t h}\left(j_{c}\right)$ curve to the right, while for $T<10 \mathrm{~K}$ - to the left. This produces the same relative shifts as the $T$-dependence of $j_{c}$, and hence does not change the conclusions of the previous paragraph. 